\title{
Technologische Ausstattung virtueller Teams
}

In diesem Kapitel sollen die benötigte technologische Ausstattung in Form von Software sowie die notwendige Technologie für Videokonferenzen im Homeoffice behandelt werden. Das Kapitel schließt mit Empfehlungen zur Einrichtung des Homeoffice und einem Exkurs zu VR-Meetings.

\subsection{Technologische Ausstattung für virtuelle Teams}

Die digitale Kommunikation und die Zusammenarbeit über Software sind essentiell für virtuelle Teams. Generell werden für eine sinnvolle Zusammenarbeit ausgewählte Software-Tools benötigt, die nachfolgend aufgelistet sind.

- Software zur Kommunikation und Zusammenarbeit (vgl. Abschn. 3.2/3.3) wie

- Dokumentenspeicherung (ownCloud oder OneDrive),

- E-Mails (Microsoft oder Open Xchange),

- Chat (Teams oder RocketChat)

- Bearbeitung von Dokumenten (Office365 oder OnlyOffice)

- Software zur Aufgaben- und Vorgangsverfolgung (Jira oder OTRS) sowie zur Wissensdokumentation (vgl. Abschn. 3.2/3.3 - Confluence oder Media Wiki)

- Möglichkeiten für virtuelle Meetings (vgl. Abschn. 3.4 - Zoom oder Jitsi)

Dies stellt die Grundausstattung dar, wobei diese mit zusätzlicher Software für spezifische Unternehmensprozesse wie Zeiterfassung, Rechnungsstellung etc. beliebig erweitert werden kann. Ich möchte mich in diesem Essential auf die Kernbereiche, also die genannten Software-Tools zur Zusammenarbeit, fokussieren. 
Empfehlenswert ist, dass diese Software-Tools gemeinsame Schnittstellen bilden, damit die Arbeit noch effizienter wird. Beispielsweise bietet es sich an, große Dokumente nicht als E-Mail-Anhang, sondern über einen Link auf die Filesharing-Lösung (Datenspeicherlösung) zu versenden oder bei Einladung zu einem Termin im Kalender direkt einen Einladungslink für ein Online-Meeting zu erstellen. Diese Integration ist nicht einfach, sondern vergleichsweise komplex, steigert jedoch die Arbeitseffizienz deutlich. Daher sollten Sie entweder eigenes Fachpersonal aufbauen oder einen zuverlässigen Provider beauftragen, damit diese Lösungen für Sie und ihre Mitarbeiter eine Hilfe und kein Frustpotenzial darstellen (vgl. Abb. 3.1).

Andere Software-Tools, z. B. zur digitalen Angebotserstellung oder Tools zur Erzeugung von Rechnungen, können als sogenannte Stand-Alone-Lösungen genutzt werden. Das bedeutet in diesem Fall, dass die Software-Lösung nicht mit einer anderen Software zusammenarbeitet oder kommuniziert. Dies ist bei einzelnen Anwendungen nicht problematisch, allerdings gerade bei der kritischen Informationsinfrastruktur nicht effizient.

Sie können die genannten Software-Tools durch kommerzielle Software, z. B. von Microsoft, oder durch freie Open-Source-Software (ownCloud,

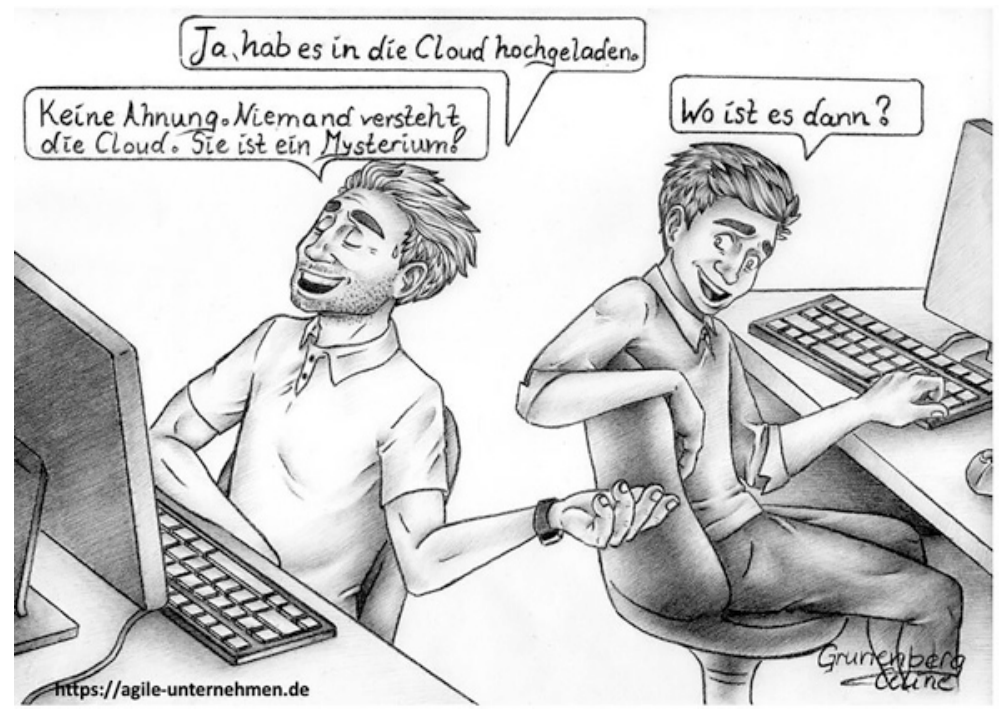

Abb. 3.1 Cloud-Anwendungen sind oft nicht trivial, so dass es sich empfiehlt, Fachpersonal einzustellen 
Open Xchange) abbilden. Im Folgenden werden beide Varianten als kompletter Software-Stack vorgestellt. Ein Software-Stack ist eine Menge von aufeinander aufbauenden Software-Tools, die gemeinsam die Ausführung einer übergreifenden Anwendung unterstützen. Die Komponenten des Software-Stacks arbeiten Hand in Hand und erreichen damit eine höhere Effizienz als das bei der Anschaffung von Einzelkomponenten der Fall ist (bspw. durch die automatische Übernahme von Daten und die sich daraus ergebende Zeitersparnis sowie durch Mehrwertfunktionen).

Merkbox Unabhängig davon, ob Sie kommerzielle oder freie Open-Source-Software verwenden - Sie müssen diese durch geeignetes IT-Personal betreiben, was zusätzliche Kosten verursacht. Alternativ können Sie den Betrieb durch einen IT-Anbieter durchführen lassen. Wichtig ist, dass Sie dies neben den Anschaffungskosten ins Budget mit einplanen (vgl. Lindner und Leyh 2019).

\subsection{Kommerzielle Software}

Die aktuell bekanntesten kommerziellen Softwarelösungen zur Kommunikation und zur Zusammenarbeit sind Microsoft Office 365 und Teams, zur Verfolgung von Aufgaben und Vorgängen auch Atlassian Jira sowie diverse Ticketsysteme.

Ich möchte in diesem Fall meine Darstellung und die entsprechenden Erklärungen auf die führenden Anbieter beschränken, da diese oft ganze Software-Stacks anbieten (Kombination aus mehreren Software-Produkten). Natürlich sollten Sie auch Alternativen berücksichtigen.

Merkbox Die Vorteile von kommerzieller Software sind oft eine leichtere Anwendung und ein entsprechendes Design. Nachteile bestehen im Bereich Datenschutz und in der hohen Abhängigkeit von einem Anbieter (engl. Vendor Lock-In).

\section{Software zur Kommunikation und zur Zusammenarbeit}

In diesem Abschnitt stelle ich die von Microsoft angebotenen Produkte zur Kommunikation und zur Zusammenarbeit von virtuellen Teams vor. Die Produkte Microsoft Office 365 und Teams kombinieren verschiedene Online-Services mit der klassischen Desktop-Office-Software, wobei Office 365 in unterschiedlichem Leistungsumfang verfügbar ist. Die Webanwendungen sind per Browser über 
das Internet und daher unabhängig vom Betriebssystem des Computers nutzbar. Office 365 beinhaltet im Kern Anwendungen wie:

- Schreibprogramm (Word),

- Tabellen (Excel),

- Präsentationen (PowerPoint),

- Notizen (OneNote),

- Videokonferenz und Chatsystem (Skype),

- E-Mail (Outlook) und

- Filesharing (OneDrive).

Dies sind die Kernbestandteile, die durch Services wie Sharepoint und weitere Komponenten ergänzt werden können. Die Abb. 3.2 zeigt die Nutzung von Office Word im Browser. Im Softwareprodukt Microsoft Teams werden die Komponenten um weitere wichtige Funktionen zur Kollaboration erweitert. Das

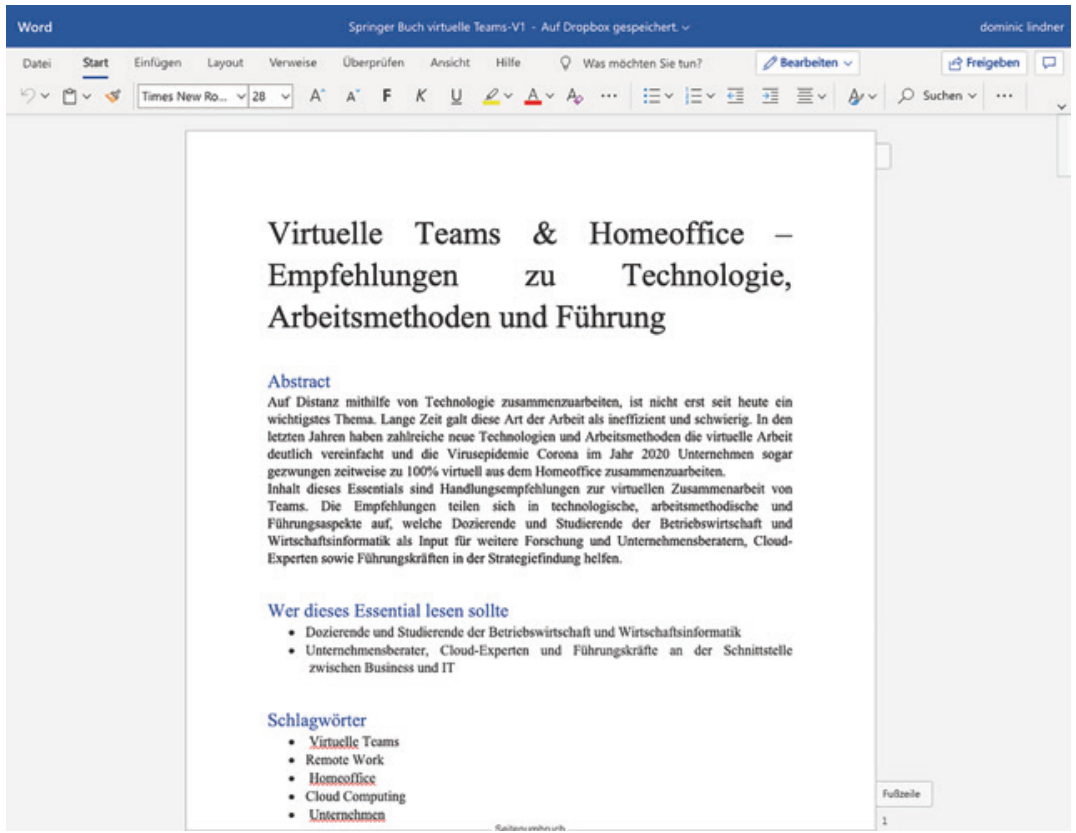

Abb. 3.2 Word in der Browserversion mit OneDrive 
Produkt ,Teams‘ ist noch relativ neu (2017) und enthält zusätzliche Meeting-, Nachrichten- und Aufgabenverwaltungsfunktionen (Erläuterung im nächsten Abschnitt durch Jira). Die Pakete können nur über Microsoft und Partnerfirmen bezogen werden und die Kosten liegen bei mindestens 10 EUR pro Nutzer pro Monat.

Weiterhin sind E-Mail und Chatfunktion erforderlich für die Kommunikation. Diese werden von Microsoft in der Regel mitgeliefert. Chatmessenger machen die Kommunikation schneller, übersichtlicher und sind weniger formell. In Kombination mit E-Mails stellen sie daher ein wichtiges Element für Unternehmen dar. Im Kontext von Microsoft werden zumeist Skype und Teams genutzt. Außerhalb von Microsoft sind Open-Source-Alternativen (z. B. RocketChat, vgl. Abschn. 3.3) weit verbreitet, die ich in diesem Kapitel noch vorgestellt werden.

\section{Software zur Vorgangs- und zur Aufgabenverwaltung}

Neben der Kommunikation und der Zusammenarbeit gilt es, die Aufgabenerledigung sicherzustellen und $\mathrm{zu}$ überwachen, welche Aufgaben erledigt worden sind. Es gibt zwei Arten der Aufgabenerledigung in virtuellen Teams: die eher kreative Aufgabenerledigung durch Teams (z. B. bei Projekten wie der Entwicklung einer App) und die prozessbasierte Aufgabenerledigung (z. B. im Kundensupport oder beim Betrieb einer Anwendung).

\section{Teambasierte Aufgabenverwaltung: Boards und Wissensdokumentation}

Speziell in der IT ist als Produkt für das Vorgangs- und Aufgabentracking Jira (Atlassian) weit verbreitet. Es wird oft gemeinsam mit dem Produkt Confluence zur Dokumentation von Wissen verwendet. Wichtig in der virtuellen Teamarbeit sind die Visualisierung und die Verteilung von Aufgaben über Ansichten wie anhand des in Abb. 3.3 gezeigten Boards. Auch ich arbeite mit einen solchen Board, um die Arbeit meiner Teams effizient organisieren zu können. Selbst dieses Essential habe ich über ein solches Software-Tool organisiert.

Ob mit einer simplen To-Do-Liste oder mit einem umfassenden Projektboard jedes Mitglied eines virtuellen Teams sollte immer wissen, woran der andere jeweils aktuell arbeitet. Der Sinn des Vorgangs- und Aufgabentrackings ist zum einen, dass nichts vergessen wird, und zum anderen, dass das Wichtigste zuerst erledigt wird. Auch für die Teamleitung und das Management kann so sichergestellt werden, dass trotz der Distanz jeder an der richtigen Sache zur richtigen Zeit arbeitet. Die Kosten für Jira beginnen ab 10 EUR pro Nutzer pro Monat, wobei das beliebig erweitert werden kann. Alternativen sind Meistertask, Trello und Microsoft Teams. 


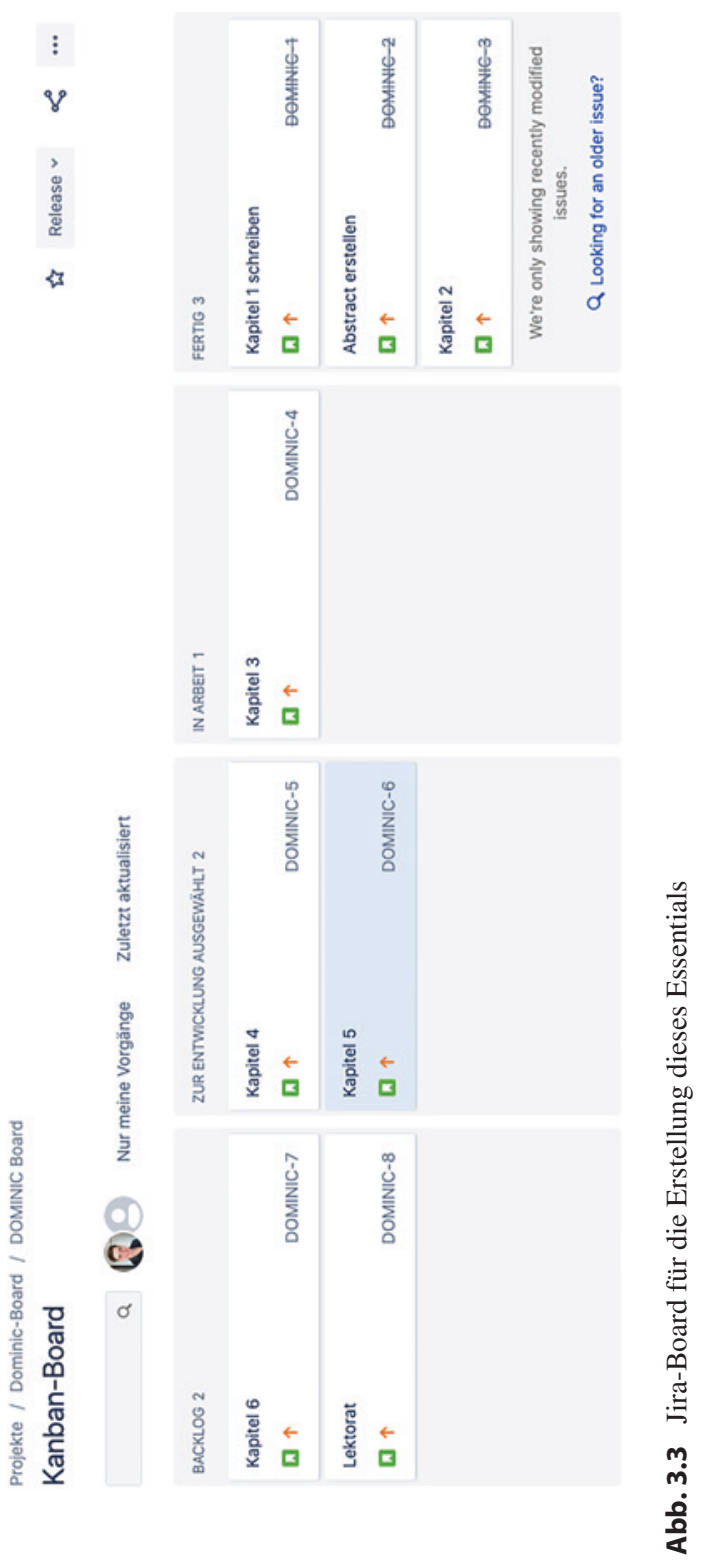


Merkbox Das gezeigte Kanban-Board (Abb. 3.3) ist grob eine horizontale Abbildung einer klassischen To-Do-Liste (engl. Aufgabenliste). Der Workflow (engl. Arbeitsfluss) kann damit jedoch besser eingesehen, überwacht und optimiert werden. Kanban-Boards gelten als moderne Form der Arbeitsvisualisierung und werden im nächsten Kap. 4 näher erläutert.

Darüber hinaus ist die Dokumentation von Wissen im Unternehmen von wesentlicher Bedeutung. Vorteile entsprechender Software-Tools zur Dokumentation sind die Transparenz, die Aktualität, die Auffindbarkeit, die Suchfunktion und die Integration mit anderer Software. Eine weit verbreitete Lösung im kommerziellen Bereich ist Confluence von Atlassian. Confluence ist für das Wissensmanagement im Unternehmen gedacht und sehr leicht bedienbar (vgl. Abb. 3.4). Ebenfalls hilfreich sind die Möglichkeiten, sich nur Änderungen in großen Dokumenten anzuzeigen zu lassen, und die Integration in die Aufgabenverfolgungssoftware Jira, sodass relevante Dokumente mit der jeweiligen Aufgabe verknüpft sind.

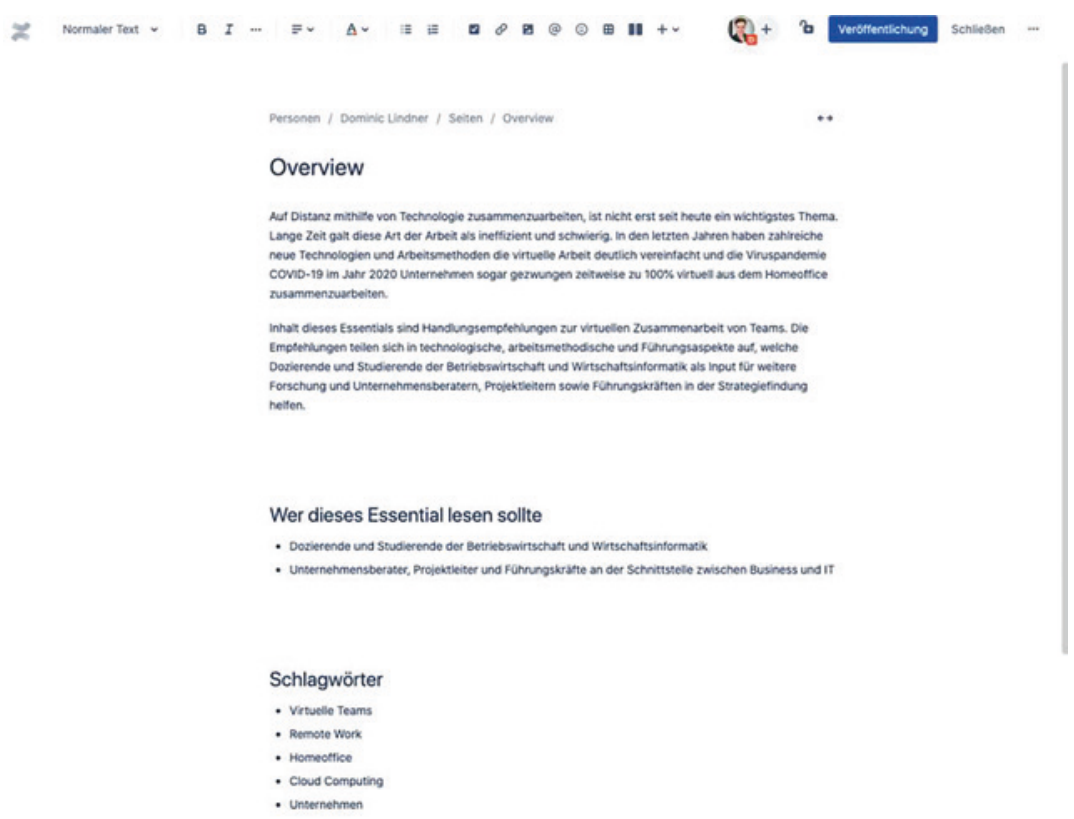

Abb. 3.4 Abbildung dieses Essentials in Confluence 


\section{Prozessbasierte Aufgabenverwaltung: Ticketsysteme}

Eine weitere Möglichkeit, speziell prozessbasierte Aufgaben zu verwalten, bieten Ticketsysteme. Ein Ticketsystem ist eine Software für den Empfang, die Klassifizierung, die Verteilung und die Bearbeitung von Anfragen.

Prozessbasiert bedeutet, dass eine Aufgabe durch ein spezielles Event wie eine E-Mail, ein IT-System oder einen Anruf eines Kunden ausgelöst wird. Das Team reagiert also auf konkrete Anfragen. Ein Beispiel ist der Kundensupport oder der Betrieb von Software. Die Kosten für Ticketsysteme im kommerziellen Bereich starten bereits bei 30 EUR im Monat, außerdem gibt es spezielle nutzerbasierte Tarife für 10 EUR pro Nutzer pro Monat.

Die Vorteile sind, dass keine Nachricht verlorengeht und jederzeit ein Gesamtüberblick über die zu bearbeitenden Aufgaben sowie eine entsprechende Kommunikation möglich sind. In der Abb. 3.5 ist ein Beispiel anhand von Freshdesk zur Bearbeitung aller Anfragen für die Erstellung dieses Essentials dargestellt. Auch ich arbeite mit einem Ticketsystem, um meine Kommunikation effizient organisieren zu können. Sie sehen hier, welche Person aktuell an welchem Ticket arbeitet und wie der aktuelle Stand der Bearbeitung ist. Außerdem können Tickets problemlos an andere Teammitglieder weitergegeben werden. Im nächsten Kap. 4 wird die tägliche Zusammenarbeit mit Ticketsystemen näher erläutert. Weitere Alternativen zu Freshdesk finden sich eher im Open-Source-Bereich (vgl. Abschn. 3.3) oder im Plugin Jira Service Desk von Atlassian.

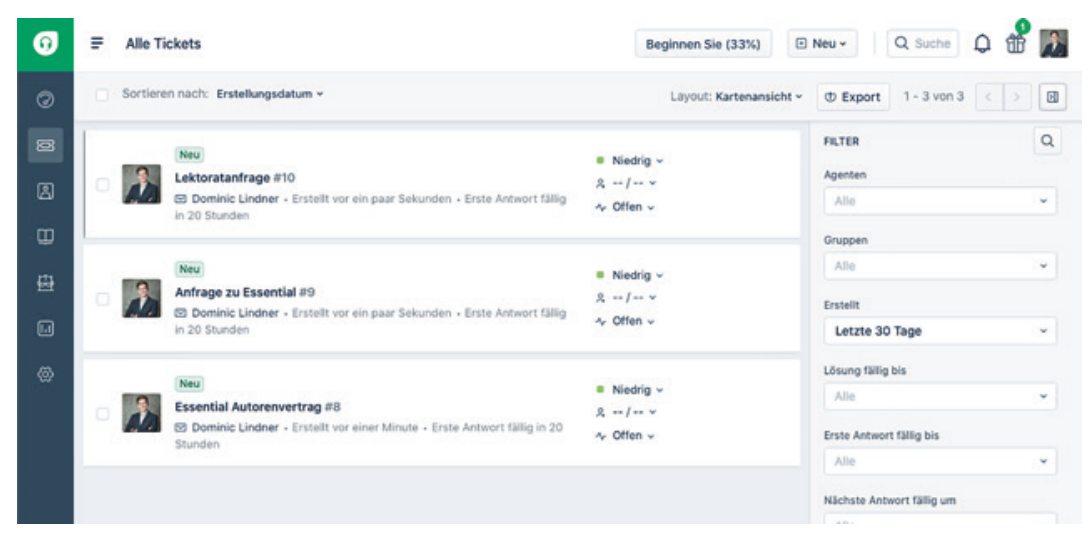

Abb. 3.5 Aufgabenverwaltung mithilfe eines Ticketsystems 


\subsection{Open-Source-Software}

Für nahezu jede Aufgabe gibt es freie, sogenannte Open-Source-Software, die hinsichtlich ihrer Leistung oft nicht hinter kommerziellen Anwendungen zurücksteht. Eine Open-Source-Software ist eine Software, deren Quellcode frei verfügbar über das Internet heruntergeladen und die im Rahmen von Open-Source-Lizenzmodellen unentgeltlich genutzt werden kann. Durch die Veröffentlichung des Quellcodes kann der Nutzer nachvollziehen, dass Daten nicht ohne seine Genehmigung verwendet werden (vgl. Lindner et al. 2019).

Auch für virtuelle Teams können Open-Source-Software-Komponenten verwendet werden. Sie können diese Lösungen selbst betreiben (auf eigenen Servern - engl. On-Premise) oder bei einem Anbieter des Vertrauens als Software-as-a-Service-Lösung (engl. SaaS) oder aber sie zu sogenannten Managed/Full-Service-Tarifen anfordern. Häufig ist die sogenannte CommunityVersion der Open-Source-Provider kostenlos. Für weitere Funktionen und für Unterstützung im Betrieb kann ein sogenannter Enterprise-Support gebucht werden. Speziell bei den Kernfunktionen ist dieser Support sinnvoll und kostet meist pro Komponente eine jährliche Gebühr, die sich in der Regel in einem ähnlichen Preisbereich wie die kommerziellen Anbieter bewegt.

Merkbox Der Vorteil der Nutzung von Open Source besteht darin, die Kontrolle und Souveränität über die eigenen Daten zu behalten. Nachteile können aus einer manchmal erschwerten Administration entstehen.

\section{Software zur Kommunikation und zur Zusammenarbeit}

Ich möchte Ihnen im Folgenden eine Alternative zur kommerziellen Software auf Basis der Open-Source-Anbieter vorstellen, die mir bekannt sind. Gemeinsam mit weiteren Open-Source-Experten arbeite ich aktuell an einem solchen Stack mit.

Als Basis nutze ich eine Filesharing-Lösung (Datenspeicherlösung), um die Daten sicher zu speichern. Der Unterschied zur kommerziellen Variante ist, dass sich die Daten nicht auf Servern wie dem von Microsoft in den USA befinden und somit die Sicherheit der Unternehmensdaten vollkommen gewährleistet werden kann. Ich verwende in diesem Beispiel ownCloud (Abb. 3.6), das Sie entweder selbst betreiben oder als Service auf https://owncloud.online beziehen können. Die Community-Version von ownCloud kann kostenlos verwendet werden. Dieses Essential habe ich sicher in einer von mir betriebenen ownCloud 


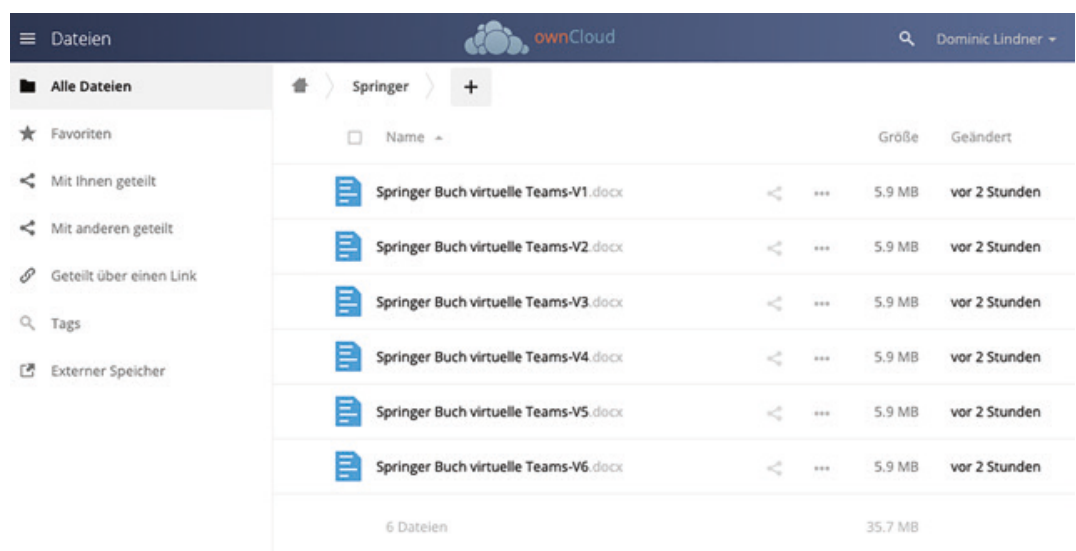

Abb. 3.6 ownCloud in der Browserversion

auf einem Raspberry-Pi-Server gespeichert und konnte so jederzeit darauf zugreifen.

Analog zu den Zusatzfunktionen von Office 365 können weitere Open-Source-Versionen bezogen werden, die mit ownCloud funktionieren und sich integrieren lassen. Diese sind:

- Schreibprogramm, Tabellen und Präsentationen (Collabora oder Onlyoffice),

- Notizen (Joplin),

- Videokonferenz (Jitsi oder Kopano),

- E-Mail (Open XChange)

- Chatsystem (RocketChat)

- Wissensdokumentation (MediaWiki)

- Ticketsysteme (OTRS und OSTicket),

- Virtuelle Aufgabenverteilung (Open Office oder Kanboard) und

- Filesharing (ownCloud).

Ein wichtiger Faktor ist die Möglichkeit, die verschiedenen Komponenten sinnvoll miteinander verknüpfen $\mathrm{zu}$ können. Oft weisen die genannten Open-Source-Anbieter schon eine Grundaffinität zueinander auf: Beispielsweise funktioniert ownCloud in der Regel ohne große Anstrengungen mit Collabora (Abb. 3.7). An diesem Essential habe ich dank eines Online-Office-Programms problemlos von jedem internetfähigen Gerät aus weiterarbeiten können. 


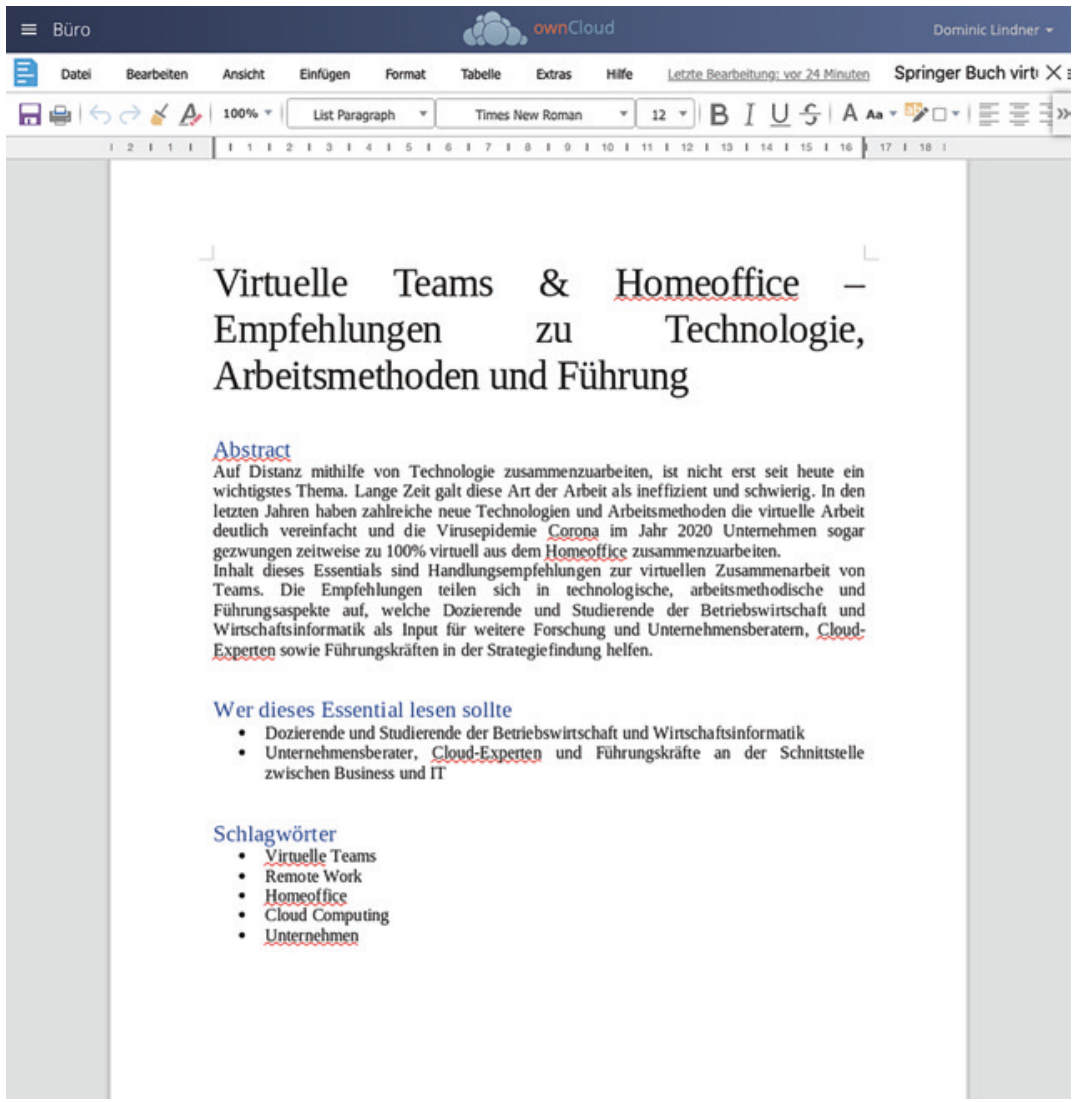

Abb.3.7 Abbildung eines Dokuments in der Open-Source-Software ownCloud mit Collabora

Merkbox In diesem Bereich scheint mir Open Source hinsichtlich des Funktionsumfangs genauso stark wie kommerzielle Lösungen. Aufgrund der Souveränität über die Daten würde ich Open Source bevorzugen.

Weitere notwendige Elemente sind Chat- und E-Mailsysteme. Open XChange ist eine freie Software mit zahlreichen Funktionen für den Versand von E-Mails. Es kann auf eigenen Servern betrieben werden und garantiert, ähnlich wie ownCloud, die Souveränität über die eigenen Daten. 


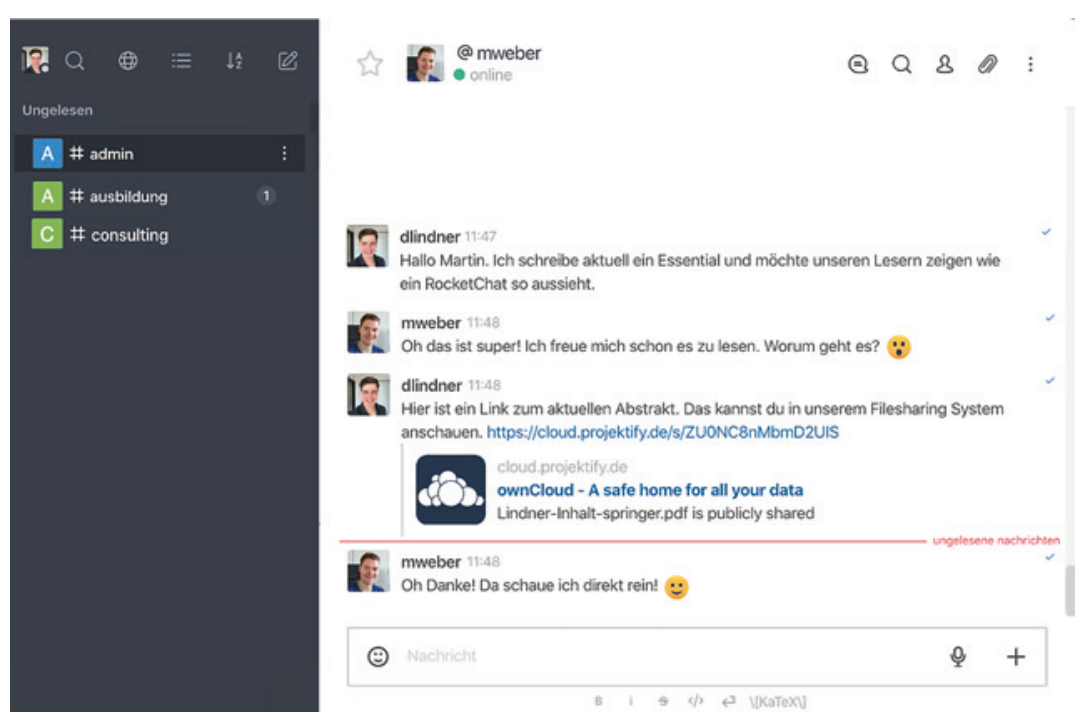

Abb. 3.8 RocketChat als Screenshot in der Community-Edition im Chat von projektify.de

Ein weiteres wichtiges Tool zur Kommunikation ist ein Chatsystem. Hier ist aktuell vor allem RocketChat (vgl. Abb. 3.8) aufgrund der einfachen Integrierbarkeit in andere Softwarekomponenten, der vielen Funktionen und der sicheren Einrichtung ein oft verwendetes Software-Tool im Open-Source-Bereich.

Team- und prozessbasierte Aufgabenverwaltung: Boards und Ticketsysteme sowie Wissensdokumentation

Auch für die Verwaltung von team- und prozessbasierten Aufgaben gibt es zahlreiche Open-Source-Alternativen. Im Folgenden sollen zwei solcher Software-Tools sowie eines für die Wissensdokumentation erläutert werden.

- Kanboard (teambasiert)

- OTRS (prozessbasiert)

- Media Wiki (Wissensdokumentation)

Kanboard (vgl. Abb. 3.9) bietet als freie Software die Möglichkeit, Arbeit übersichtlich und transparent darzustellen. Es eignet sich für teambasierte 


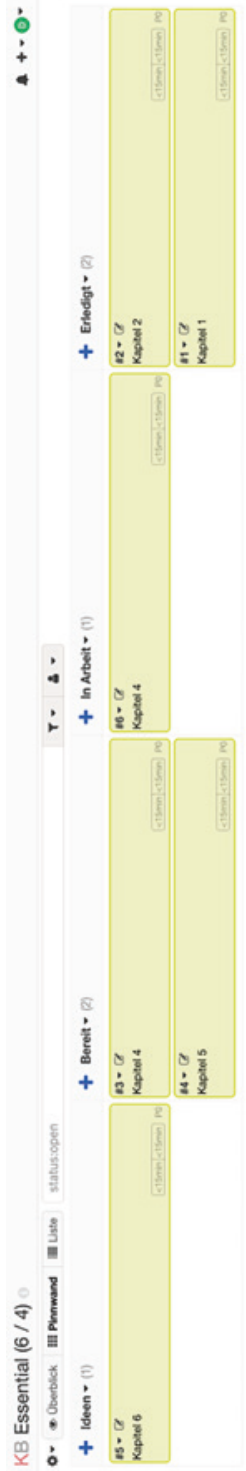

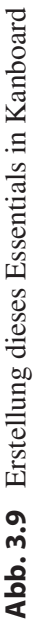


Arbeit. Ähnlich wie bei Jira können verschiedene Projekte angelegt und verwaltet werden. Mein Fazit ist, dass die Software grundlegende Funktionen zur Verwaltung von Aufgaben bietet und dauerhaft weiterentwickelt wird. Insgesamt zeigt Kanboard den Fortschritt und welche Aufgaben gerade von wem erledigt werden. Alternativen sind Restyaboard, Open Project oder Redmine.

OTRS ist ein webbasiertes Ticketsystem (vgl. Abb. 3.10). Es eignet sich für prozessbasierte Arbeit, um jede Art von Anfragen, z. B. E-Mails, Störungsmeldungen und Telefon sowie formularbasierte Anfragen strukturiert zu erfassen, $\mathrm{zu}$ klassifizieren, zu speichern und weiterzuverarbeiten. Das System bietet umfassende Möglichkeiten und sorgt mit detaillierten Reports für Revisionssicherheit (Archivierung). OTRS ist aktuell eine der führenden Lösungen in diesem Bereich, die laut Heise (2013) von $60 \%$ der deutschen DAX-Konzerne genutzt wird. Alternativen sind OSTicket und Zammad.

Merkbox Im Bereich der teambasierten Aufgabenverwaltung würde ich die kommerziellen Software-Tools aktuell als etwas fortschrittlicher betrachten, während im Bereich der prozessgesteuerten Aufgabenverwaltung die freien Open-Source-Lösungen im Vorteil sind.

Wikipedia ist Ihnen sicher bekannt - die Grundlage (Software) dieser Online-Dokumentation ist ein Media Wiki. Generell sind Wikis gut anpassbar und erfüllen die Mehrzahl der Zwecke, die für die gemeinsame Dokumentation von Wissen erforderlich sind. Sie verhindern außerdem das Anlegen von mehreren Versionen, da ein Dokument von allen Mitarbeitern bearbeitet werden und versioniert abgerufen werden kann. Abb. 3.11 zeigt dieses Essential in Form eines Media Wiki.

\section{Sinnvolle Verknüpfung der einzelnen Software-Komponenten}

Ein wichtiger Aspekt ist die Verknüpfung der einzelnen Software-Produkte. Dies geschieht in der Regel über bestimmte Schnittstellen (engl. API). Welche Schnittstellen unterstützt werden, kann auf der Produktseite der Hersteller nachgelesen werden. Auch kommerzielle Software-Tools wie Jira verfügen über eine API und können mit Open-Source-Anwendungen verknüpft werden. Oft empfehlen die Hersteller selbst bestimmte Software-Komponenten und sind bereits mit automatischer Integration ausgestattet, z. B. ownCloud/Collabora, Jira/RocketChat oder Open Xchange/ownCloud. 


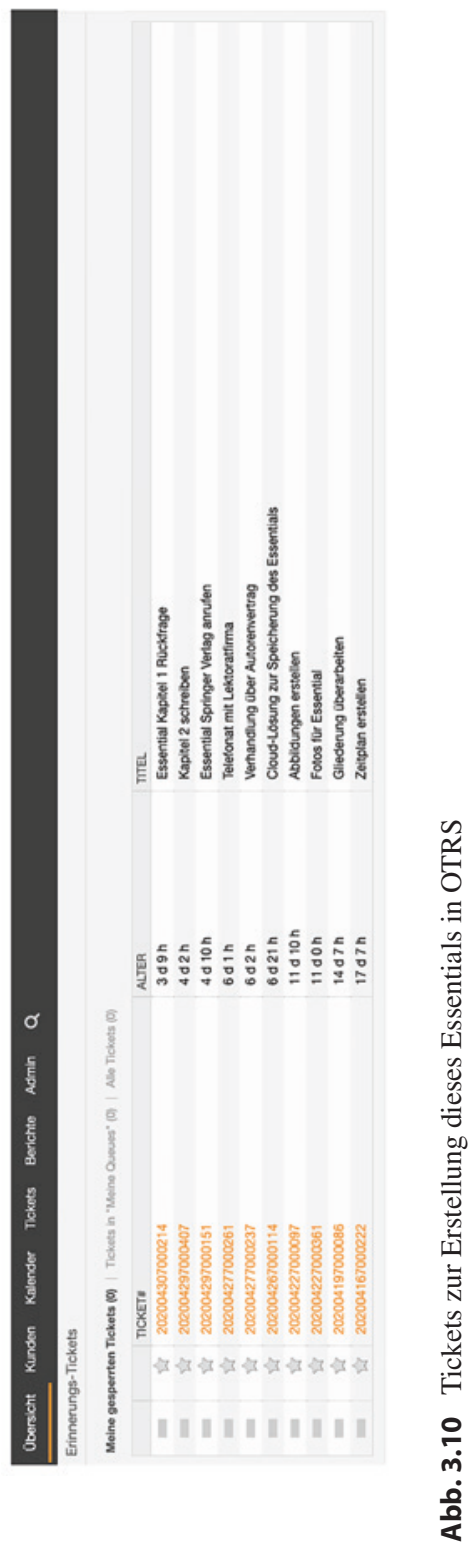




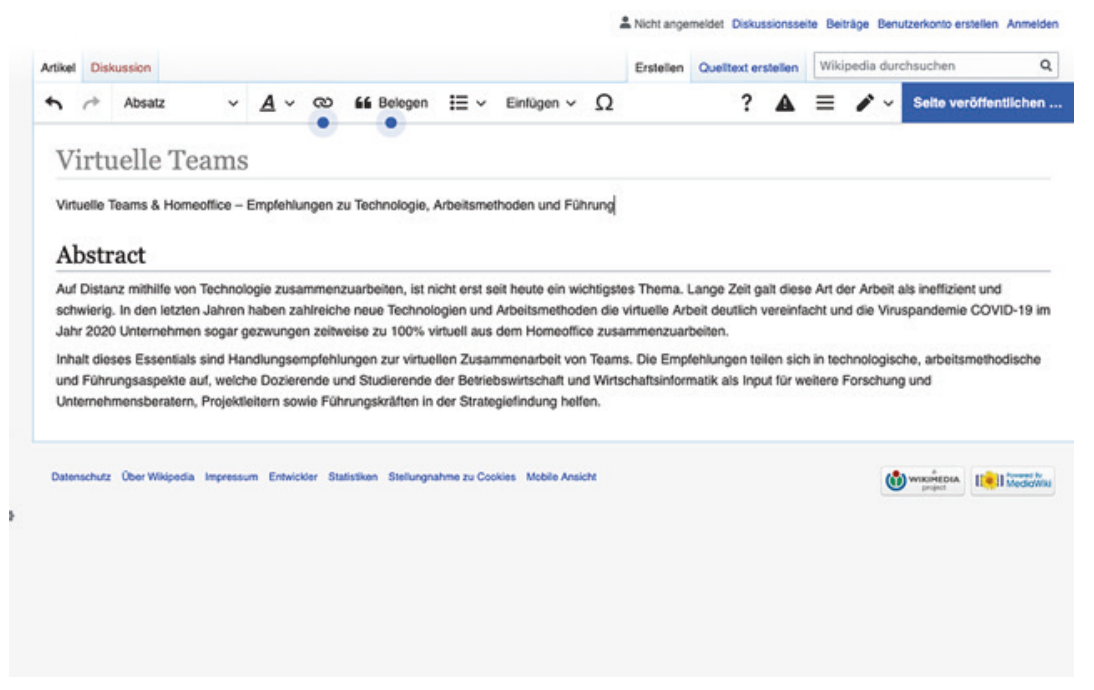

Abb. 3.11 Dieses Essential in Form eines Media Wiki

Entscheidend ist schließlich, welche toolübergreifenden Anwendungsfälle Sie abbilden sollten, um die Arbeit zu erleichtern. Am besten fragen Sie dazu Ihre Mitarbeiter. Im Folgenden sind einige sinnvolle Anwendungsfälle aus meinem Berufsalltag stichpunktartig aufgeführt:

- Dokumente können direkt in der Filesharing-Lösung bearbeitet und geteilt werden (Online Office Funktion).

- E-Mail-Anhänge werden direkt als Link aus der Filesharing-Lösung erstellt.

- Dokumente und Aufgaben (z. B. in Jira) können miteinander verknüpft werden.

- Im Chat kann auf vorhandene Dokumente und Aufgaben verwiesen werden.

- Automatische Erstellung eines virtuellen Meetings bei Einladung von Teilnehmern im Kalender

- Automatische Abwesenheitsnachricht bei E-Mails bei der Eintragung von Urlaub im Kalender

- Zum Abschluss noch eine Verknüpfung mit der Zeitbuchung, die in diesem Kapitel nicht näher erläutert wurde: Schnelle Zeitbuchung nach Abschließen einer Aufgabe oder eines Tickets mit Übernahme der wesentlichen Informationen, z. B. Betreff (vgl. Kap. 4). 


\subsection{Virtuelle Meetings und Einrichtung im Homeoffice}

Video- und Telefonkonferenzen sind in vielen Unternehmen schon lange üblich. Vor allem die Einsparungen hinsichtlich Reisezeit und Kosten sind Vorteile, die dafür sprechen. Ich möchte in diesem Kapitel einige Empfehlungen für die Durchführung von Video- und Telefonkonferenzen geben. Diese betreffen die Software, das Mikrofon sowie die Einrichtung des eigenen Homeoffice.

\section{Virtuelle Meetings}

Mittlerweile hat die Zahl der Video- und Telefonkonferenzen aufgrund von COVID-19 und der damit verbundenen Änderungen in der Arbeitsweise deutlich zugenommen. Zu Beginn der Ausgangsbeschränkungen im März 2020 ist es aufgrund der massiven Nutzung sogar vermehrt zu Ausfällen gekommen. Aktueller Marktführer für die entsprechende Software ist Zoom mit einer sicheren und verschlüsselten Videolösung. Auch bieten Videokonferenz-Lösungen die Möglichkeit, per Telefon teilzunehmen oder Dokumente gemeinsam zu betrachten. Die Preise für Zoom beginnen bei 14 EUR pro Monat pro Nutzer. Alternativen sind im kommerziellen Bereich Skype, Goto Meeting, Webex und im Open-Source-Bereich Kopano und Jitsi (vgl. Abb. 3.12).

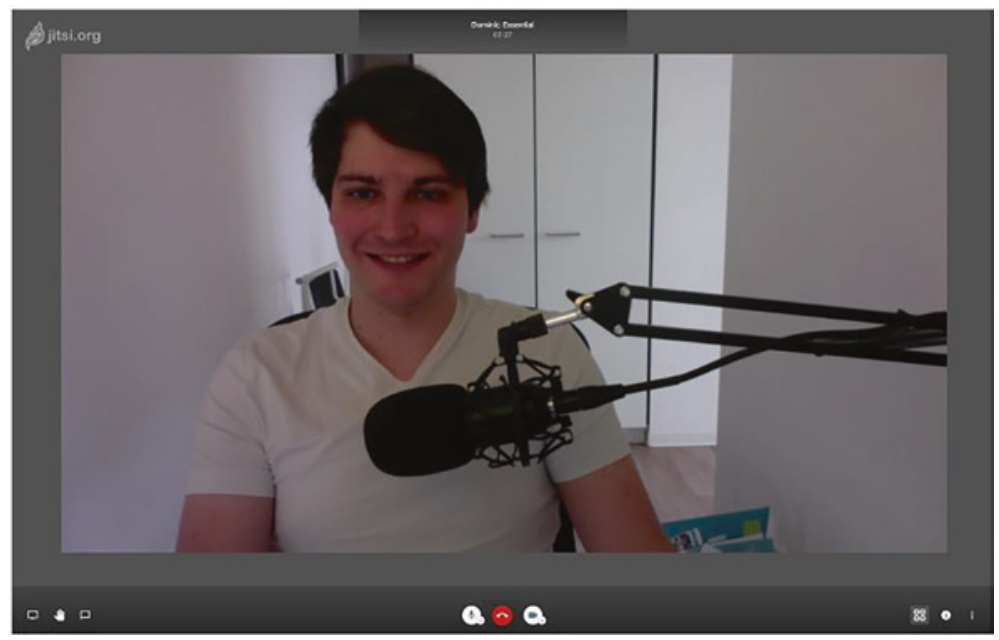

Abb. 3.12 Screenshot aus der Software Jitsi in meinem Homeoffice 
Ist Ihnen in Abb. 3.12 mein Mikrofon aufgefallen? Es handelt sich um ein professionelles Studiomikrofon für die virtuellen Konferenzen. Für die professionelle Nutzung ist meines Erachtens die im PC verbaute Hardware nicht ausreichend. Achten Sie auf einen guten Sound für das Headset am Telefon und am Computer: Je besser Sie zu verstehen und zu sehen sind, desto komfortabler verlaufen Ihre Verhandlungen im virtuellen Raum.

Im Rahmen der virtuellen Arbeit fallen, wie bereits erwähnt, viele Konferenzen und Telefonate an. Leisten Sie sich daher eine Webcam für mind. 150 EUR und nutzen Sie ein professionelles Mikrofon (vgl. Abb. 3.13). Für die Telefonie verwende ich ein Bluetooth-Headset (zwischen 100 und 300 EUR) mit aktiver Rauschunterdrückung, das sogar die Geräusche der Umgebung für den Anrufer reduziert (z. B. wenn auf dem Balkon Vögel oder Autos zu hören sind). Für Videokonferenzen habe ich ein Studiomikrofon mit Audio-Interface, das eine klare Stimme gewährleistet (ab 200 EUR im Set). Natürlich müssen Sie nicht ebenso viel investieren, doch Sie sollten zumindest ein qualitativ hochwertiges Mikrofon oder Headset besitzen.

\section{Einrichtung im Homeoffice}

Die Ausstattung des eigenen Homeoffice ist für die Effizienz der Arbeit von wesentlicher Bedeutung. Zwar ist es im Rahmen des Essentials nicht möglich,
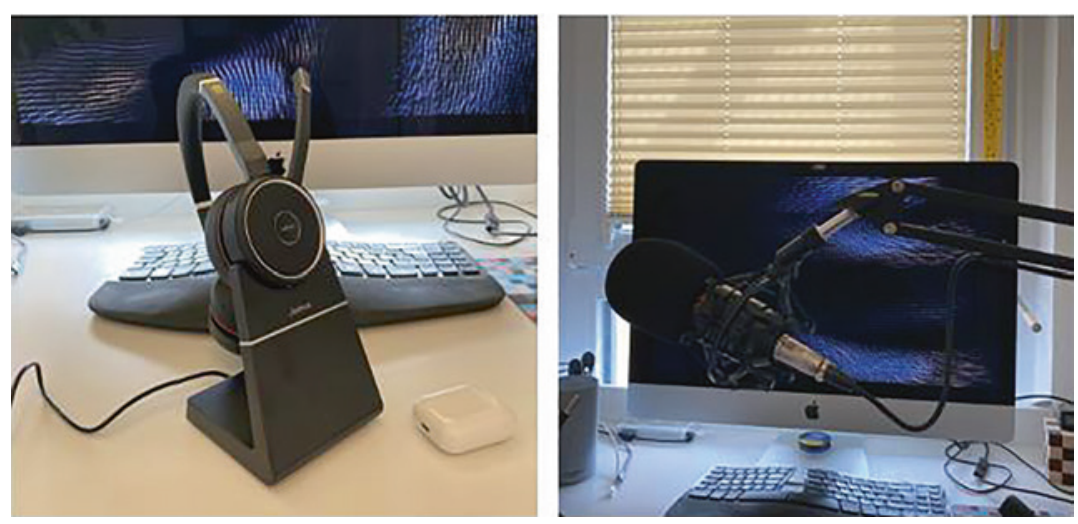

Abb. 3.13 Meine Ausstattung besteht aus einem Jabra Evolve 75 Headset für die Telefonie und einem Kondensator Mikrofon (T-Bone 400) mit Steinberg HR12 Audiointerface für die Videokonferenz 
detaillierte Angaben in Bezug auf Ergonomie oder Möbel zu machen, doch möchte ich einige grundlegende Empfehlungen in Form einer Checkliste geben. Am Anfang oder bei gelegentlichem Homeoffice mag es vorkommen, dass am Küchentisch oder auf dem Sofa gearbeitet wird. Dies ist in Bezug auf die Arbeitseffizienz und auch mit Blick auf die Gesundheit nicht zu empfehlen (z. B. kann das zu Rückenproblemen führen). Meine Empfehlung: Richten Sie sich ein geeignetes Homeoffice ein. Aus meiner Erfahrung sind notwendig:

- Bildschirm: Sorgen Sie für einen ausreichend großen Bildschirm oder zwei und einen Abstand von mind. $50 \mathrm{~cm}$ zum Bildschirm.

- Computer: Sorgen Sie für einen ausreichend starken Desktop-PC oder Laptop mit Docking-Station. Ich selbst verwende einen Apple iMac.

- Stuhl und Schreibtisch: Investieren Sie auf jeden Fall in einen bequemen Stuhl mit einer entsprechenden Ausrichtung auf langes Sitzen, denn Sie sitzen unter Umständen $40 \mathrm{~h}$ pro Woche. Auch ein hochfahrbarer Schreibtisch ist auf Dauer eine Alternative.

- Gesundheit: Ich empfehle, bei Maus und Tastatur auf ein ergonomisches Modell zurückzugreifen. Ihre Hände sind essenziell für Ihre Arbeit. Schonen Sie diese. Auch ein paar Halsbonbons schonen Ihre Stimme nach langen Telefonkonferenzen.

- Licht: Achten Sie auf ein ausreichend gutes Licht durch ein Fenster tagsüber und abends in Form einer Schreibtischlampe. Licht im Rücken oder direkt auf den Bildschirm schädigt die Augen. Ich nutze beispielsweise Lampen von Philips Hue, die verschiedene Lichteinstellungen ermöglichen.

- Standort: Am besten ist ein separates Zimmer oder eine sehr ruhige Nische in der Wohnung. Bei Kindern empfiehlt sich auf jeden Fall eine abschließbare Tür. Essen Sie nicht am PC. Nutzen Sie die Mittagspause für einen Ortswechsel und führen Sie eine wenig intensive Telefonkonferenz gegebenenfalls auch auf den Balkon oder im Wohnzimmer durch.

- Dekoration: Sie sollten sich wohlfühlen am Schreibtisch. Durch Pflanzen und persönliche Gegenstände wirkt die Umgebung ansprechender.

\subsection{Exkurs: VR-Meetings}

Aufgrund von COVID-19 sind sehr viele Menschen gezwungen, von Zuhause aus $\mathrm{zu}$ arbeiten und auch fast die gesamte Freizeit dort $\mathrm{zu}$ verbringen (Stand April 2020). Dies kann im Job wie auch privat zur Isolation führen. Ich habe dazu im 
April 2020 intensiv überlegt, wie sich die Nähe zum Team durch die Technologie Virtual Reality (VR) fördern lässt. Virtual Reality (Virtuelle Realität, VR) ist eine computergenerierte Wirklichkeit mit Bild (3D) und in vielen Fällen auch mit Ton (Gabler 2020).

Ich habe mir mit einigen Kollegen eine VR-Brille (ca. 120 EUR) gekauft (vgl. Abb. 3.14). Wir wollten ausprobieren, ob wir uns mit dieser Technologie noch effizienter als per Video treffen und Konzepte sowie arbeitsrelevante Themen sinnvoll diskutieren können.

Mein Fazit ist, dass es eine spannende Alternative $\mathrm{zu}$ herkömmlichen Meetings vor allem bei räumlich weit distanziert arbeitenden Teams (Europa und USA) mit geringen Möglichkeiten für persönliche Treffen ist. Allerdings besteht noch deutlicher Entwicklungsbedarf, um diese Technologie in einem Unternehmen sinnvoll einsetzen zu können (Stand April 2020). Aktuell fehlen noch neben Datenschutzkonzepten vor allem sinnvolle virtuelle Meetingräume und die passenden Business-Avatare (virtuelle Darstellung eines Menschen). Ich würde mir wünschen, dass ich meine Avatare schnell und mit wenigen Klicks an meine Meetings anpassen kann. Im Innovationsprojekt gehören laut Erwartungshaltung nur Jeans und Hemd, während ich für das anschließende Management-Meeting mit Hemd und Jackett auftreten möchte.

Abb. 3.14 Ich in meinem VR-Meeting mit Kollegen auf dem Balkon

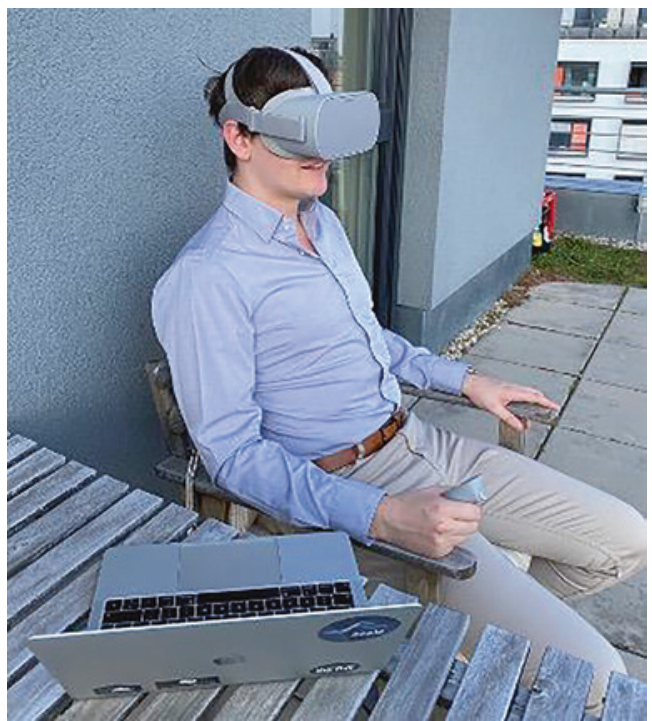




\subsection{Fazit}

Sie werden festgestellt haben, dass zahlreiche Software-Tools notwendig sind, damit virtuelle Teams sinnvoll zusammenarbeiten können. Möglich ist, zwischen kommerzieller und freier Open-Source-Software zu wählen. Dabei ist die Entscheidung meines Erachtens in jeder Hinsicht von der Unternehmensstrategie abhängig. Wichtig ist, dass Sie in beiden Fällen die Software sinnvoll miteinander interagieren lassen. Dazu können Sie entweder alle Komponenten vom gleichen Anbieter kaufen (was nicht immer sinnvoll oder möglich ist) oder die Open-Source-Komponenten entsprechend integrieren. Diese Integration ist entscheidend, ob eine Softwarelandschaft effizient ist oder nicht. Beispielsweise könnten zu große E-Mail-Anhänge direkt als Link in die E-Mail eingefügt und Dokumente per Klick im Browser unmittelbar bearbeitet werden. Es kann Mitarbeiter demotivieren, wenn Technologie nicht ausreichend funktioniert oder sie nicht die volle Effizienz eines virtuellen Teams erreichen, wie in Kap. 2 beschrieben (Abb. 3.15). Vor allem im Bereich der Kommunikationssoftware

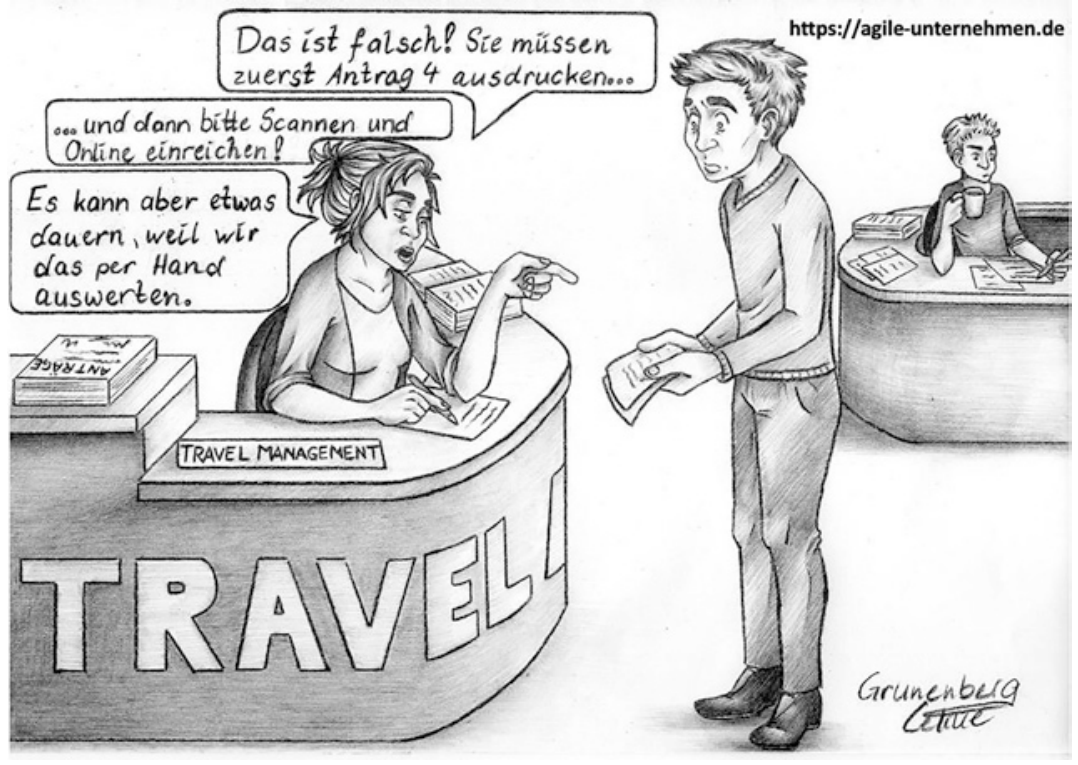

Abb. 3.15 Wichtig ist die passende Integration der Software 
sehe ich große Vorteile bei der Open-Source-Software, die mittlerweile sehr ausgereift ist. Aufholbedarf besteht dagegen im Bereich des Vorgangs- und des Aufgabentrackings. Hier liegen für mich aktuell die kommerziellen Lösungen sowie OTRS (Open Source) vorne.

Die beste Software nützt nichts, wenn sich der Mensch vor dem Bildschirm (also Sie) nicht wohl fühlt. Aus diesem Grund empfehle ich Ihnen, sich Gedanken über die Einrichtung des Homeoffice zu machen und sich ein professionelles Mikrofon für Telefon- und Videokonferenzen anzuschaffen. Entsprechende Empfehlungen aus meiner Praxiserfahrung habe ich für Sie zusammengestellt. Am Ende möchte ich Ihnen zusätzlich empfehlen, die Augen nach neuen Möglichkeiten und Trends offen $\mathrm{zu}$ halten, wie sie z. B. mit VR-Meetings bestehen. 